\title{
Tomato Hybrid CTH1: A High Yielding Hybrid with Better Shelf Life
}

\author{
L. Pugalendhi*, V. Rajashree and M. Prabhu \\ Horticultural College and Research Institute, Tamil Nadu Agricultural University, \\ Coimbatore - 641 003, India \\ *Corresponding author
}

\section{A B S T R A C T}

\begin{tabular}{l} 
Ke y w o r d s \\
$\begin{array}{l}\text { CTH1, } \mathrm{F}_{1} \text { hybrid, } \\
\text { Leaf curl virus, } \\
\text { Moderate resistance } \\
\text { and Tomato }\end{array}$ \\
\hline Article Info \\
$\begin{array}{l}\text { Accepted: } \\
18 \text { August } 2020 \\
\text { Available Online: } \\
\text { 10 September } 2020\end{array}$ \\
\hline
\end{tabular}

CTH 1 Tomato is a $F_{1}$ hybrid of LE 1226 X LE 1249. It was developed by the Department of Vegetable Science, Horticultural College and Research Institute, Tamil Nadu Agricultural University, Coimbatore. Fruits are flat round with green shoulder during ripening and turned into red colour at full ripening with an average fruit weight of $75.40 \mathrm{~g}$. The fruits of the hybrid could be harvested for 20-22 times in five months duration. Due to the long harvesting period, the single plant yields 36 fruits with an average yield of 2.94 $\mathrm{kg} /$ plant. The hybrid culture CTH 1 proved its superiority with an yield of $92.3 \mathrm{t} / \mathrm{ha}$ which was 27.31 per cent increase over the check TNAU tomato hybrid CO 3 and 40.91 per cent over another check Lakshmi (C) respectively. It showed consistent performance in Multi location Trials (14 Nos) and Adaptive Research Trials (107 Nos). The plants can be grown at a spacing of $60 \times 45 \mathrm{~cm}$ under irrigated condition. The plants should be staked. It can be successfully grown in June -July and Dec - Jan under irrigated conditions. This hybrid is having better shelf life compared with other commercial hybrids. This hybrid can be recommended for cultivation in the districts of Coimbatore, Cuddalore, Dharamapuri, Dindigul, Erode, Kancheepuram, Karur, Krishnagiri, Madurai, Namakkal, Pudukottai, Ramanathapuram, Salem, Thanjavur, Thirunelveli, Thiruvannamalai, Theni, Thoothukudi, Tirupur, Trichy, Vellore and Villupuram of Tamil Nadu.

\section{Introduction}

Tomato (Solanum lycopersicum L.) is one of the important vegetables grown in the world. Tomato originated in Peru Equador region of Latin America and was introduced by Portuguese in India. It is cultivated worldwide in variety of climatic conditions including sub tropics and tropics. Among the vegetables tomato occupies fourth position in area and second position in production in India. In India, tomato occupies a larger area of 0.79 million hectare with an annual production of 19.76 million tonnes (Anonymous, 2018). It is universally treated as a "protective food" and it is also an excellent vegetable for processing. The fruits are consumed raw, cooked or processed as juice, ketchup, sauce, paste, puree etc. (Rani et al., 2009). Being a good source of minerals, acids, vitamins (A, $\mathrm{B}$ and $\mathrm{C}$ ) and lycopene it has high nutritive value (Sumathi et al., 2006 and Kavitha et al., 2007). The scenario of tomato production in India has tremendously changed with 
increasing popularity of hybrids (Pugalendhi, 1986). The growers, consumers and processing industries are with pressing demand to evolve high yielding hybrids with varying qualities as per local demands. Increased vigour in total yield in tomato to the extent of 300 per cent over the commercial varieties has been reported. In India, the extent of the cross over the better parent is 203 per cent (Cheema and Dhaliwal, 2004). Commercial hybrids of tomato are products of mid twentieth century though their valued performance was recognized a century and a quarter ago (Anbukkarasi et al., 2018). Hence, it is imperative to obtain hybrids which have high yielding potential along with good quality and resistance to pests and diseases. With this background a tomato $F_{1}$ hybrid CTH1 was developed and evaluated under different environments for commercial exploitation.

\section{Materials and Methods}

The present investigation was carried out at the Department of Vegetable Science, Horticultural College and Research Institute, TNAU, Coimbatore Out of 15 tomato hybrid combinations developed through Line $X$ Tester analysis (Kempthorne, 1957) by using 5 lines and 3 testers, two hybrids $\mathrm{CTH} 1$ (LE1226 x LE 1249) and CTH 2 (LE $1251 \mathrm{x}$ LE 1229) were identified with high yield. The parental lines are maintained by continuous selfing and hybrid seeds are produced by hand emasculation and crossing. The hybrids were evaluated for three seasons during 2012-14 at College orchard, Tamil Nadu Agricultural University (TNAU), Coimbatore. Based on the consistent yield and consumer preference, the hybrid $\mathrm{CTH} 1$ was identified as the best and compared with check hybrids viz., TNAU tomato hybrid $\mathrm{CO} 3$ and Lakshmi under multilocation trials (8 Nos.) at TNAU research stations during 2015-16 and 201617, adaptive research trials (107 Nos.) at farmers holdings located in 17 districts of Tamil Nadu during 2017-18, on farm trials (5 Nos.) during 2018-19, demonstration trials (2 Nos.) and all India coordinated trials (6 Nos.). The descriptors for tomato $\mathrm{F}_{1}$ hybrid $\mathrm{CTH} 1$ and its female (LE 1226) and male parents (LE 1249) are described as per descriptors of IPGRI, 1996.

The seed rate followed for tomato hybrids is $150 \mathrm{~g} / \mathrm{ha}$ and spacing adopted was $60 \times 45 \mathrm{~cm}$ (37,037 plants/ ha) The nutrients applied @ 50:250:100 NPK kg/ha, as basal and top dressing of $\mathrm{N}$ and $\mathrm{K}$ each @ $150 \mathrm{~kg} / \mathrm{ha}$ in 3 equal splits at 30, 45 and 60 days after transplanting. Yellow sticky traps @ 10 numbers were used to attract adult insects. The affected plants were uprooted and one spray of Thiamethoxam $25 \%$ WG @ $0.5 \mathrm{ml} /$ litre of water was given to control whiteflies.

Observations on yield parameters viz., number of fruits per plant, mean fruit weight, yield per plant and pest and disease incidence were recorded. The quality traits viz.,, TSS (by digital hand refractometer), and ascorbic acid were estimated as per standard procedures (AOAC,1984). The fruits were given for organoleptic evaluation by a panel of ten judges and evaluation was done as per the characteristics in the score card for organoleptic evaluation. Based on one to nine 'Hedonic Scale', the colour and appearance, flavour, texture, taste and overall acceptability were evaluated and marks awarded to the above hybrids. The mean value was reckoned as final score (Prabhu et al., 2010).

Disease scale for tomato leaf curl virus was followed as suggested by Banerjee and Kalloo (1987). 
Per cent disease incidence (PDI) for Tomato leaf curl virus

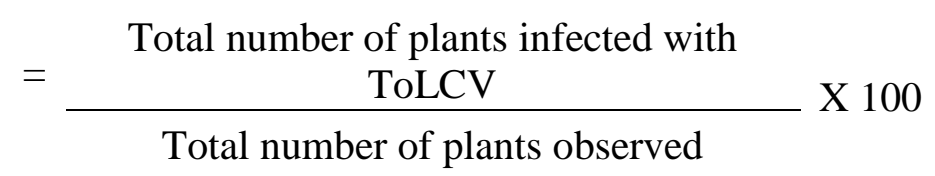

\section{Results and Discussion}

\section{Morphological characters}

Potato leaf type was observed in Tomato $F_{1}$ hybrid CTH1 and its female parent LE 1226 and standard leaf type was observed in male parent LE 1249 (Table 1). These leaf types are also reported by Bhattarai et al., (2018). Tomato $\mathrm{F}_{1}$ hybrid $\mathrm{CTH} 1$ plants are semi determinate $(100-110 \mathrm{~cm})$ and training is required at 30 days after planting. The semideterminate genotypes are equivalent to determinate ones with extended vegetative growth, which in turn impacts shoot height, number of leaves and either stem diameter or internode length. Semi-determinate plants also tend to increase the highly relevant agronomic parameter Brix $\times$ ripe yield (BRY). Water- se efficiency (WUE), evaluated either directly as dry mass produced per amount of water transpired or indirectly through $\mathrm{C}$ isotope discrimination was higher in semi-determinate genotypes. The previous experimental results shown that the increases in BRY in semi-determinate genotypes are a consequence of an improved balance between vegetative and reproductive growth, a mechanism analogous to the conversion of the overly vegetative tall cereal varieties into well-balanced semi-dwarf ones used in the green revolution (Vicente et al., 2015). The first fruit setting was observed at 65 days after planting, Fruits are flat round with five locules, green shoulder at breaker stage which turns to red colour during ripening (Table 1).

\section{Yield and quality characters}

The fruits are borne in clusters of five, with an average fruit weight of $80-100 \mathrm{~g}$ and belongs to medium maturity type. This hybrid has long harvesting period with 20-22 harvests in 160 - 165 days with a yield of 2.35 $\mathrm{kg}$ per plant (Table 1). The ascorbic acid content: is $26.13 \mathrm{mg} / 100 \mathrm{~g}$ and TSS is: $6.7^{0}$ brix and pericarp thickness is $5.84 \mathrm{~mm}$. These results clearly indicated that the tomato hybrid CTH1 is highly suitable for processing and value addition as compared with check hybrids. This character is very much useful to control market price fluctuation and losses of tomato (Tripathi et al., 2017). Similarly, the hybrid CTH1 ranked first for consumer preference followed by check hybrids TNAU tomato hybrid $\mathrm{CO} 3$ and Lakshmi (Table 2).

\section{Reaction to insect pests and diseases}

The tomato hybrid CTH1 recorded the least (9.7/ three leaves) adult whitefly population followed by Tomato hybrid CO 3 (10.7). While, Lakshmi hybrid was the most preferred with the highest population (28.5/three leaves). CTH1 maintained its superiority over check with significantly the least (8.1) number of adult thrips per three leaves. The other pests viz., tomato leaf miner, Tuta absoluta and serpentine leaf miner, Liriomyza trifolii was recorded very minimum of 4.3 and $7.8 \%$ compared to check 27.14 and $17.5 \%$ respectively. There was no incidence of fruit borer and leaf eating caterpillar damage on $\mathrm{CTH} 1$ and Tomato hybrid CO 3.

The tomato leaf curl virus disease transmitted by white fly and caused by begomovirus is a destructive disease of tomato in many parts of India. 
Table.1 Morphological descriptors of the Tomato hybrid CTH 1 and parents

\begin{tabular}{|c|c|c|c|c|}
\hline Sl.No & Plant characters & CTH 1 & Female (LE 1226) & Male (LE 1249) \\
\hline I & \multicolumn{4}{|l|}{ Growth characteristics } \\
\hline 1 & Plant height $(\mathrm{cm})$ & $100-110 \mathrm{~cm}$ & $85 \mathrm{~cm}$ & $65 \mathrm{~cm}$ \\
\hline 2 & Plant growth habit & Semi determinate & Semi determinate & Determinate \\
\hline 3 & $\begin{array}{l}\text { Number of primary } \\
\text { branches }\end{array}$ & 4 & 3 & 2 \\
\hline 4 & Leaf type & Potato leaf & Potato leaf & Standard \\
\hline 5 & Leaf colour & Green & Green & Green \\
\hline 6 & Leaf pubescence & Absent & Present & Present \\
\hline 7 & Foliage cover & Excellent & Excellent & Sparse \\
\hline 8 & Petiole pubescence & Present & Present & Present \\
\hline 9 & Stem thickness & Medium & $4.8 \mathrm{~cm}$ & $2.9 \mathrm{~cm}$ \\
\hline 10 & Stem pubescence & Sparse & Sparse & Sparse \\
\hline 11 & Stem pigmentation & No & No & No \\
\hline II & \multicolumn{4}{|c|}{ Flowering characteristics } \\
\hline 1 & Flower colour & Deep yellow & Deep yellow & Deep yellow \\
\hline 2 & Style position & $\begin{array}{l}\text { Same level as } \\
\text { stigma }\end{array}$ & $\begin{array}{l}\text { Same level as } \\
\text { stigma }\end{array}$ & $\begin{array}{l}\text { Same level as } \\
\text { stigma }\end{array}$ \\
\hline 3 & Days to first fruit set & 65 DAP & $60-65$ DAP & 62-65 DAP \\
\hline 4. & Total crop duration & $160-165$ days & 155-160 days & 140-145 days \\
\hline 5 & $\begin{array}{l}\text { Number of clusters per } \\
\text { plant }\end{array}$ & 16 & 12 & 6.2 \\
\hline 6 & $\begin{array}{l}\text { Number of flowers per } \\
\text { cluster }\end{array}$ & 5 & 6 & 4.51 \\
\hline III & \multicolumn{4}{|l|}{ Fruiting characteristics } \\
\hline 1 & Fruit size & $\begin{array}{l}\text { Medium large }(< \\
80-100 \mathrm{~g})\end{array}$ & $\begin{array}{l}\text { Medium large (80 } \\
-100 \mathrm{~g})\end{array}$ & $45-50 \mathrm{~g}$ \\
\hline 2 & Fruit shape & Flat round & Heart shaped & Flat round \\
\hline 3 & $\begin{array}{l}\text { Immature fruit skin } \\
\text { colour }\end{array}$ & Green & Light green & Dark green \\
\hline 4 & $\begin{array}{l}\text { Presence of green } \\
\text { shoulder on fruits }\end{array}$ & Present & No & Yes \\
\hline 5 & Fruit colour & $\begin{array}{l}\text { Green shoulder } \\
\text { during the breaker } \\
\text { stage. Green } \\
\text { shoulder disappears } \\
\text { at fully ripened } \\
\text { stages and red } \\
\text { colour develops. }\end{array}$ & $\begin{array}{l}\text { Light green during } \\
\text { breaker stage and } \\
\text { turn red colour } \\
\text { during maturity. }\end{array}$ & $\begin{array}{l}\text { Prominent green } \\
\text { shoulder even after } \\
\text { ripening. }\end{array}$ \\
\hline 6 & Fruit surface & Smooth & Smooth & Smooth \\
\hline 7 & Stem end fruit shape & Indented & Indented & Indented \\
\hline 8 & Blossom end fruit shape & Flat & Slightly flatted & Slightly flatted \\
\hline
\end{tabular}




\begin{tabular}{|c|c|c|c|c|}
\hline 9 & Type of fruit cracking & None & None & None \\
\hline 10 & Fruit firmness $\left(\mathrm{kg} / \mathrm{cm}^{2}\right)$ & $\begin{array}{l}\text { Semi ripening - } \\
2.76 \text { and full } \\
\text { ripened }-2.04\end{array}$ & 3.86 & 1.86 \\
\hline 11 & Pulpiness & Pulpy & Highly pulpy & Pulpy \\
\hline 12 & Seediness & Medium & Medium & Medium \\
\hline IV & \multicolumn{4}{|l|}{ Yield characteristics } \\
\hline 1 & $\begin{array}{l}\text { Number of fruits per } \\
\text { cluster }\end{array}$ & 4 & 5 & 4.1 \\
\hline 2 & No. of fruits/plant & 32.1 & 31.2 & 26.7 \\
\hline 3 & Locule number per fruit & 4 & 4 & 3.7 \\
\hline 4 & Fruit length $(\mathrm{cm})$ & 5.50 & 5.20 & 4.7 \\
\hline 5 & Fruit girth (cm) & 11.0 & 10.54 & 7.5 \\
\hline 6 & Fruit weight (g) & 88.4 & 80 & 50 \\
\hline 7 & Fruit yield per plant & 2.35 & 1.89 & 1.78 \\
\hline 8 & Fruit yield (t / ha) & 90.8 & 46.9 & 39.5 \\
\hline V & \multicolumn{4}{|l|}{ Quality characteristics } \\
\hline 1 & Pericarp thickness (mm) & 5.84 & 4.97 & 3.87 \\
\hline 2 & Total soluble solids & 6.70 & 5.67 & 4.09 \\
\hline 3 & $\begin{array}{l}\text { Ascorbic acid } \\
(\mathrm{mg} / 100 \mathrm{~g})\end{array}$ & 26.13 & 24.1 & 23.7 \\
\hline 4 & Seed shape & Ovate & Ovate & Ovate \\
\hline 5 & Seed colour & Light yellow & Light yellow & Creamy yellow \\
\hline VI & \multicolumn{4}{|c|}{ Biotic stress susceptibility } \\
\hline 1 & Leaf curl incidence $(\%)$ & $\begin{array}{l}10.5 \text { (moderately } \\
\text { resistant) }\end{array}$ & $\begin{array}{l}10.5 \text { (moderately } \\
\text { resistant) }\end{array}$ & $\begin{array}{l}11.4 \text { (moderately } \\
\text { resistant) }\end{array}$ \\
\hline 2 & Pest incidence (borer) & 6.0 & 6.4 & 7.9 \\
\hline
\end{tabular}

Table.2 Quality parameters and consumer preference of Tomato hybrid CTH 1 and check hybrids

\begin{tabular}{|c|c|c|c|c|c|c|c|c|}
\hline \multirow[t]{2}{*}{ Hybrids } & \multicolumn{3}{|c|}{ Quality parameters } & \multicolumn{5}{|c|}{ Consumer preference } \\
\hline & $\begin{array}{c}\text { Ascorbic } \\
\text { acid } \\
(\mathrm{mg} / 100 \mathrm{~g})\end{array}$ & $\begin{array}{c}\text { TSS } \\
\left({ }^{0} \mathrm{Brix}\right)\end{array}$ & $\begin{array}{c}\text { Pericarp } \\
\text { thickness } \\
\quad(\mathbf{m m})\end{array}$ & $\begin{array}{c}\text { Colour and } \\
\text { appearance }\end{array}$ & Flavour & Texture & Taste & $\begin{array}{c}\text { Overall } \\
\text { acceptability }\end{array}$ \\
\hline CTH 1 & 26.13 & 6.70 & 5.84 & 9 & 9 & 9 & 9 & 9 \\
\hline $\begin{array}{l}\text { TNAU } \\
\text { Tomato } \\
\text { hybrid CO } 3 \\
\text { (C) }\end{array}$ & 35.72 & 5.58 & 5.21 & 8 & 8 & 9 & 9 & 8.5 \\
\hline Lakshmi (C) & 28.27 & 4.52 & 3.13 & 7 & 9 & 9 & 8 & 8.25 \\
\hline Mean & 30.04 & 5.60 & 4.73 & 8 & 8.7 & 9 & 8.7 & 8.6 \\
\hline
\end{tabular}


Table.3 Screening of Tomato hybrid CTH1 and check hybrids for major insect pests and diseases during 2017-18

\begin{tabular}{|c|c|c|c|c|c|c|c|c|c|c|}
\hline \multirow[t]{2}{*}{ Hybrids } & $\begin{array}{l}\text { Fruit } \\
\text { borer }\end{array}$ & $\begin{array}{c}\text { Leaf } \\
\text { miner }\end{array}$ & $\begin{array}{l}\text { Serpentine } \\
\text { leaf miner }\end{array}$ & Thrips & Whitefly & $\begin{array}{c}\text { Damping } \\
\text { off }\end{array}$ & $\begin{array}{c}\text { Fusarium } \\
\text { wilt }\end{array}$ & $\begin{array}{l}\text { Early } \\
\text { blight }\end{array}$ & $\begin{array}{l}\text { Spotted } \\
\text { wilt } \\
\text { virus }\end{array}$ & $\begin{array}{c}\text { Tomato } \\
\text { leaf curl } \\
\text { virus }\end{array}$ \\
\hline & \multicolumn{3}{|c|}{ Pest incidence $(\%)^{*}$} & \multicolumn{2}{|c|}{$\begin{array}{c}(\mathrm{No} / \mathrm{3} \\
\text { leaves/plant })^{*}\end{array}$} & \multicolumn{5}{|c|}{ Disease incidence / intensity (\%) } \\
\hline CTH 1 & - & 4.3 & 7.8 & 8.1 & 9.7 & 5.80 & 12.00 & 5.75 & 10.50 & 10.00 \\
\hline $\begin{array}{l}\text { TNAU } \\
\text { Tomato } \\
\text { hybrid } \\
\text { CO } 3\end{array}$ & - & 4.7 & 8.2 & 9.4 & 10.7 & 6.25 & 12.21 & 6.00 & 11.55 & 11.11 \\
\hline Lakshmi & 14.98 & 27.14 & 17.5 & 24.7 & 28.5 & 8.33 & 14.33 & 7.00 & 15.33 & 24.33 \\
\hline Mean & - & 12.0 & 11.2 & 14.1 & 16.3 & 6.79 & 12.85 & 6.25 & 12.46 & 15.15 \\
\hline
\end{tabular}

Table.4 Overall performance of Tomato hybrid CTH 1 under station, multi location, adaptive and demonstration trials

\begin{tabular}{|c|c|c|c|c|c|c|c|c|c|c|}
\hline \multirow[t]{2}{*}{ Particulars } & \multirow{2}{*}{$\begin{array}{c}\text { No. } \\
\text { of } \\
\text { trials }\end{array}$} & \multicolumn{3}{|c|}{ Number of fruits / plant } & \multicolumn{3}{|c|}{$\begin{array}{l}\text { Average fruit weight } \\
\text { (g) }\end{array}$} & \multicolumn{3}{|c|}{ Mean yield / plant (kg) } \\
\hline & & CTH 1 & $\begin{array}{l}\text { TNAU } \\
\text { Tomato } \\
\text { hybrid } \\
\text { CO } 3(\mathrm{C})\end{array}$ & $\begin{array}{l}\text { Lakshmi } \\
\text { (C) }\end{array}$ & CTH 1 & $\begin{array}{l}\text { TNAU } \\
\text { Tomato } \\
\text { hybrid } \\
\text { CO 3 } \\
\text { (C) }\end{array}$ & $\begin{array}{l}\text { Lakshmi } \\
\text { (C) }\end{array}$ & CTH 1 & $\begin{array}{l}\text { TNAU } \\
\text { Tomato } \\
\text { hybrid } \\
\text { CO } 3(\mathrm{C})\end{array}$ & $\begin{array}{l}\text { Lakshmi } \\
\text { (C) }\end{array}$ \\
\hline $\begin{array}{l}\text { HC \& RI, } \\
\text { Coimbatore }\end{array}$ & 3 & 38.1 & 29.4 & 30.8 & 77.3 & 61.7 & 63.9 & 2.73 & 2.27 & 2.18 \\
\hline $\begin{array}{l}\text { Large Scale } \\
\text { demonstration } \\
\text { at HC \& RI } \\
\text { Coimbatore }\end{array}$ & 1 & 38.0 & 31.8 & 28.9 & 78.0 & 61.3 & 64.8 & 3.56 & 1.98 & 2.0 \\
\hline MLT I & 8 & 31.0 & 28.1 & 28.4 & 67.4 & 52.8 & 57.2 & 2.40 & 1.50 & 1.60 \\
\hline MLT II & 6 & 39.8 & 34.0 & 36.8 & 71.1 & 64.2 & 62.4 & 2.51 & 1.70 & 1.80 \\
\hline ART & 107 & 35.8 & 31.9 & 32.9 & 81.1 & 69.2 & 71.1 & 2.97 & 2.36 & 2.37 \\
\hline $\begin{array}{l}\text { Large Scale } \\
\text { demonstration } \\
\text { at Farmers } \\
\text { Field } \\
\text { (Sattakalpudur) }\end{array}$ & 1 & 36.2 & 31.2 & 30.4 & 77.5 & 65.3 & 67.8 & 3.45 & 1.98 & 2.10 \\
\hline Mean & 126 & 36.48 & 31.07 & 31.37 & 75.40 & 62.42 & 64.53 & 2.94 & 1.97 & 2.01 \\
\hline $\begin{array}{l}\text { Yield increase } \\
\text { over check }\end{array}$ & \multicolumn{7}{|c|}{$27.31 \%$ over Tomato hybrid CO 3 (C) } & \multicolumn{3}{|c|}{$\begin{array}{l}\text { 40.91\% over } \\
\text { Lakshmi (C) }\end{array}$} \\
\hline
\end{tabular}


Table.5 Mean performance of the Tomato hybrid CTH 1 under adaptive research trials (2016-17)

\begin{tabular}{|c|c|c|c|c|c|c|c|c|c|c|}
\hline \multirow[t]{2}{*}{ S. No } & \multirow{2}{*}{$\begin{array}{c}\text { Name of the } \\
\text { District \& number } \\
\text { of Blocks }\end{array}$} & \multicolumn{3}{|c|}{ Number of fruits per plant } & \multicolumn{3}{|c|}{ Average fruit weight (g) } & \multicolumn{3}{|c|}{ Fruit yield/plant (kg) } \\
\hline & & CTH 1 & $\begin{array}{l}\text { TNAU } \\
\text { Tomato } \\
\text { hybrid } \\
\text { CO } 3\end{array}$ & Lakshmi & CTH 1 & $\begin{array}{c}\text { TNAU } \\
\text { Tomato } \\
\text { hybrid } \\
\text { CO } 3\end{array}$ & Lakshmi & CTH 1 & $\begin{array}{l}\text { TNAU } \\
\text { Tomato } \\
\text { hybrid } \\
\text { CO } 3\end{array}$ & Lakshmi \\
\hline 1 & Coimbatore (10) & 35.7 & 32.0 & 33.5 & 75.9 & 64.2 & 67.3 & 2.4 & 1.9 & 2.10 \\
\hline 2 & Cuddalore (10) & 36.7 & 32.0 & 32.8 & 74.8 & 63.8 & 64.7 & 2.31 & 1.88 & 1.84 \\
\hline 3 & Dharmapuri (10) & 25.0 & 26.0 & 26.0 & 60.0 & 65.0 & 65.0 & 4.00 & 3.75 & 3.00 \\
\hline 4 & Dindigul (10) & 36.8 & 32.4 & 32.8 & 74.7 & 64.7 & 64.6 & 2.30 & 1.85 & 1.90 \\
\hline 5 & Erode (10) & 19.0 & 16.7 & 21.1 & 117.2 & 106.7 & 87.2 & 2.20 & 1.80 & 1.60 \\
\hline 6 & Karur (1) & 80.0 & 70.0 & 75.0 & 100.0 & 70.0 & 80.0 & 8.0 & 5.0 & 6.00 \\
\hline 7 & Namakkal (6) & 36.9 & 33.7 & 33.0 & 75.6 & 66.3 & 66.7 & 2.3 & 1.80 & 1.90 \\
\hline 8 & Pudukottai (5) & 21.4 & 14.6 & 15.4 & 162.8 & 89.0 & 127.8 & 4.0 & 2.76 & 3.18 \\
\hline 9 & Ramanad (2) & 37.5 & 33.5 & 32.0 & 74.0 & 66.2 & 64.5 & 2.40 & 2.10 & 1.80 \\
\hline 10 & Thanjavur (1) & 45.0 & 42.0 & 48.0 & 80.0 & 75.0 & 69.0 & 3.60 & 3.20 & 3.30 \\
\hline 11 & Thoothukudi (2) & 27.5 & 24.0 & 21.5 & 41.5 & 40.0 & 43.0 & 1.08 & 0.96 & 0.91 \\
\hline 12 & Krishnagiri (5) & 36.0 & 32.60 & 33.60 & 74.6 & 64.8 & 66.3 & 2.40 & 1.77 & 2.03 \\
\hline 13 & Salem (10) & 33.6 & 29.30 & 30.3 & 83.1 & 72.7 & 68.29 & 2.26 & 1.82 & 1.85 \\
\hline 14 & Madurai (10) & 35.2 & 30.42 & 30.95 & 83.9 & 66.7 & 71.7 & 2.43 & 1.88 & 2.10 \\
\hline 15 & Theni (5) & 37.6 & 32.7 & 34.16 & 74.7 & 64.9 & 65.8 & 2.35 & 1.93 & 1.90 \\
\hline 16 & Trichy (5) & 28.3 & 27.83 & 26.5 & 67.0 & 70.3 & 71.0 & 4.08 & 3.62 & 3.11 \\
\hline 17 & Vellore (5) & 37.3 & 32.9 & 32.4 & 73.8 & 65.4 & 65.2 & 2.31 & 2.02 & 1.79 \\
\hline & Mean (107) & 35.8 & 31.9 & 32.9 & 81.1 & 69.2 & 71.1 & 2.97 & 2.36 & 2.37 \\
\hline
\end{tabular}

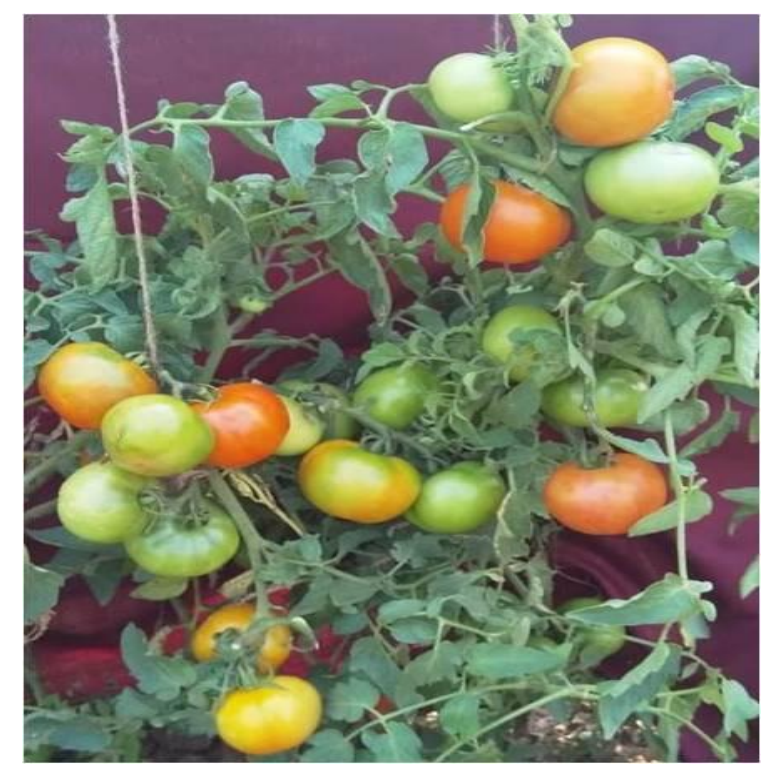

Tomato Hybrid CTH 1 
The results of the present study revealed that the tomato culture $\mathrm{CTH} 1$ recorded less tomato leaf curl virus (10.00 PDI) incidence compared to the check hybrid TNAU Tomato hybrid CO 3 (11.11 PDI) and Lakshmi (24.33 PDI) (Table 3). Similar findings in screening of tomato hybrids against tomato leaf curl virus resistance were reported by Babu et al., (2017).

\section{Performance of tomato hybrids under different field trials}

The tomato hybrid CTH1 recorded the highest yield under station trials at Coimbatore for three seasons over check hybrids with a yield of $95.8 \mathrm{t} /$ ha (Table 4). Similarly, the same recorded the maximum yield of $73.1 \mathrm{t} / \mathrm{ha}$, $78.9 \mathrm{t} / \mathrm{ha}$ and $95.6 \mathrm{t} / \mathrm{ha}$ in MLT 1, MLT 2 and ART respectively. Whereas, the check hybrid (Lakshmi) recorded minimum yield of 57.5 t/ha, 66.0 t/ha and 65.9 t/ha in MLT 1, MLT 2 and ART respectively. This Tomato hybrid CTH 1 recorded $27.31 \%$ increase in yield over Co3 and $40.91 \%$ increase over Lakshmi. The large scale demonstration was conducted in farmers field at Sattakal pudur and these results also revealed that Tomato hybrid CTH 1 recorded maximum yield of $94.5 \mathrm{t} / \mathrm{ha}$, however, the check varieties viz., Tomato hybrid CO 3 and Lakshmi recorded the yield of $73.4 \mathrm{t} / \mathrm{ha}$ and $69.3 \mathrm{t} / \mathrm{ha}$ respectively (Table 4).

In conclusion, the tomato hybrid $\mathrm{CTH} 1$ has recorded the yield of $92.3 \mathrm{t} / \mathrm{ha}$ which is 27.31 per cent higher over TNAU Tomato hybrid CO 3 and 40.91 per cent highest over hybrid Lakshmi. The tomato hybrid CTH 1 has recommended for Coimbatore, Cuddalore, Dharamapuri, Dindigul, Erode, Kancheepuram, Karur, Krishnagiri, Madurai, Namakkal, Pudukottai, Ramanathapuram, Salem, Thanjur, Thirunelveli, Thiruvannamalai, Theni, Thoothukudi, Tirupur, Trichy, Vellore and Villupuram, districts of Tamil Nadu (Table 5) and under irrigated conditions during June-July and December - January.

\section{References}

Anbukkarasi, V., M. Prabu, P. Paramaguru, L. Pugalendhi and P. Jeyakumar.2018. Effect of nutrients and Biostimulants on growth, yield and quality of Tomato (Solanum lycopersicon). Int.J.Curr. Microbiol.App.Sci., Special Issue-6: 4955.

Anon. 2018. Horticultural Statistics at a Glance 2018 Ministry of Agriculture \& Farmers' Welfare Government of India, New Delhi. pp.10.

AOAC, 1984. Official Methods of Analysis (14th Ed.), Washington DC, USA.

Babu,M. R., R. V. S. K. Reddy, K. R. Reddy, A. S. Rani and P. Saidaiah.2018. Genetic improvement for yield, quality and leaf curl virus resistance in tomato (Solanum lycopersicum L.) J. Pharmacognosy Phytochemistry, SP1:1048-1055

Banerjee, M. K. and G. Kalloo, 1987. Inheritance of tomato leaf curl virus resistance in Lycopersicon hirsutum $\mathrm{f}$. glabratum. Euphytica., 36:581-584.

Bhattarai, K., S. Sharma and D. R. Panthee, 2018. Diversity among modern tomato genotypes at different levels in freshmarket breeding. Int. J. Agronomy, 115.

Cheema, D.S. and M.S. Dhaliwal, (2004). Hybrid Tomato Breeding. In : Hybrid vegetable development (Eds. P. K. Singh, S. K. Dasgupta and S.K. Tripathi). The Haworth Press, Philadelpia, USA.

IPGRI, 1996. Descriptors for tomato (Lycopersicon spp). IPGRI, Rome, Italy.

Kavitha, M., S. Natarajan, L. Pugalendhi, N. Meenakshi. 2007. Influence of shade on 
physiological and yield parameters of tomato (Lycopersicon esculentum Mill.). Orissa J. Hort.. 8136(2):1-8.

Kempthorne O., 1957. An Introduction to Genetic Statistics, John Wiley \& Sons, New York, NY, USA..

Prabhu, M., S. Praneetha and S. Natarajan, 2010. Organoleptic evaluation of brinjal genotypes. Agric. Sci. Digest., 30 (1): $73-74$.

Pugalendhi, L. 1986. Studies on heterosis and combining ability in tomato, M.Sc. Thesis, Tamil Nadu Agricultural University, Madurai (India).

Rani, C. I., D. Veeraragavathatham and M. Prabhu, 2009. Heterosis studies in tomato (Lycopersicon esculentum Mill.) hybrids for root and biochemical characters for root knot nematode resistance. Advances in Environmental Biology, 3(2): 120-124.

Srinivasan, J., A. Vijayakumar, P. Srimathi, L. Pugalendhi. 2011. Effect of crossing period and crossing techniques on production of quality seed in tomato hybrid COTH 2. IUP J. Genetics \& Evolution. 4(1): 29-36.

Sumathi, T., V. Ponnuswami, S.Natarajan, and L. Pugalendhi. 2006. Evaluation of tomato varieties for yield and quality parameters. ATSH, 33:20-21.

Tripathi, S. P., R. P. Patel, S. P. S. Somvanshi, H. P. Singh and B. Dubey. 2017. Impact of value added tomato based product for income generation of farm women. Plant Archives, 17(2): 1329-1331.

Vicente, M. H., A. Zsogon, A. F. L. Sa, R. V. Ribeiro, L. E. P. Peres, 2015. Semideterminate growth habit adjusts the vegetative to reproductive balance and increases productivity and water-use efficiency in tomato (Solanum lycopersicum). Plant Physiol., 177:1119.

\section{How to cite this article:}

Pugalendhi, L., V. Rajashree and Prabhu, M. 2020. Tomato Hybrid CTH1: A High Yielding Hybrid with Better Shelf Life. Int.J.Curr.Microbiol.App.Sci. 9(09): 2574-2582. doi: https://doi.org/10.20546/ijcmas.2020.909.322 